\title{
Modelo Preditivo para o Problema da Dieta
}

\author{
Glauber S. Santos \\ Departamento de Computação \\ Universidade Federal de Ouro Preto \\ Ouro Preto, Brasil \\ glauber.santos@aluno.ufop.edu.br
}

\author{
Alan R. R. Freitas \\ Departamento de Computação \\ Universidade Federal de Ouro Preto \\ Ouro Preto, Brasil \\ alandefreitas@ufop.edu.br
}

\begin{abstract}
Resumo - Há um esforço constante para desenvolver e aprimorar estratégias ao lidar com o problema clássico da dieta. Modelos matemáticos e técnicas de programação têm sido desenvolvidos para geração de menus restritivos. No entanto, uma nova tendência ainda pouco abordada, no âmbito computacional, é a nutrição comportamental. Essa abordagem voltada para o aconselhamento nutricional, mostra-se mais eficaz que dietas restritivas comuns, por se adaptar aos hábitos e preferências dos usuários buscando o equilíbrio entre liberdade, saúde e sabor. Portanto, visando contribuir para uma alimentação menos restritiva e mais intuitiva, neste artigo, propomos um modelo de otimização interativo, resolvido por meio de um algoritmo evolutivo. O usuário interage após cada refeição e os dados dos alimentos ingeridos são usados para prever preferências e reequilibrar o cardápio das próximas refeições, caso seja necessário. Por fim, o modelo proposto demonstrou ser capaz de balancear com sucesso refeições com um consumo calórico até 5 vezes mais acurado que o esperado.

Palavras-chave: Problema da dieta. Otimização Dinâmica. Filtro Colaborativo.
\end{abstract}

\begin{abstract}
Since the classic diet planning problem, several mathematical models and programming techniques have been shown to generate menus following several restrictions. However, a subject still little addressed, in the computational environment, is behavioral nutrition. This scientific approach focused on nutritional counseling proves to be more effective than common restrictive diets, by adapting to the habits and preferences of users seeking balance among freedom, health and taste. Therefore, aiming to contribute to a less restrictive and more intuitive diet, this paper proposes an interactive optimization model, resolved through an evolutionary algorithm. The user interacts with the system after each meal and nutrition facts are used to predict preferences and re-balance the goal for next meals. Finally, the proposed model shows success in re-balancing meals with a target caloric consumption up to 5 times more accurate than expected.
\end{abstract}

Keywords: Diet Problem. Dynamic Optimization. Colaborative Filter.

Os autores agradecem a Universidade Federal de Ouro Preto (UFOP), ao Conselho Nacional de Desenvolvimento Científico e Tecnológico (CNPq 402956/2016-8), e à Fundação de Amparo à Pesquisa do estado de Minas Gerais (FAPEMIG - APQ-00040-14) pelo apoio ao desenvolvimento deste trabalho.

\section{INTRODUÇÃO}

O problema da dieta original (PD) [1] tinha por desafio gerar um plano alimentar com o menor custo possível. Desde então, modelos e técnicas para resolver o PD e suas variações foram criados considerando modelos restritivos. A linha do tempo [2] relacionada ao PD pode ser resumida em 3 grandes perspectivas históricas. A primeira geração do planejamento de menus automatizados se concentrou na minimização dos custos. A segunda buscou a inclusão de preferências alimentares, pré-definidas, no cardápio, e a terceira geração foi baseada em desenvolver dietas conforme as demandas específicas das pessoas.

Estudos recentes, relacionados a uma boa alimentação, mostram que dietas restritivas não promovem mudanças comportamentais e nem tornam as pessoas mais saudáveis [3; 4]. Há evidências de que, em indivíduos obesos, as restrições alimentares frequentes parecem ser parcialmente responsáveis pelas falhas e rejeições aos tratamentos da obesidade [5]. Neste contexto, uma nova abordagem científica é a nutrição comportamental, centrada no aconselhamento nutricional, que difere da visão restritiva sobre o que é saudável [6].

Ainda hoje, os sistemas de recomendação são estudados como uma solução eficaz para ajudar os usuários na escolha dos alimentos. Este é um domínio complexo, que traz desafios, não somente pela quantidade de alimentos e porções, mas devido à possibilidade de combiná-los, aumentando sua complexidade exponencialmente [7].

Os trabalhos de [8] e [9] indicam que as novas e futuras demandas de pesquisas em PD estão relacionadas à coleta de informações do usuário, novos dados nutricionais de alimentos e receitas, apoio na mudança comportamental e geração de cardápios com possíveis substituições.

Em 2016, foi apresentada uma variação do PD, visando minimizar o desvio calórico, ao invés de minimizar o custo. Os cardápios eram divididos em horários e possuíam restrições para as categorias de alimentos (café da manhã, lanches, almoço e jantar) [10]. Para isto, o autor propôs uma formulação de programação linear inteira mista e um algoritmo de evolução diferencial para resolver o problema. No ano seguinte, este mesmo autor propôs um segundo modelo de programação quadrática inteira mista resolvido por Algoritmo Genético [11]. Neste último, foram comparados os resultados obtidos pelos dois trabalhos, demonstrando que o segundo, 
com formulação combinatória junto a uma meta-heurística bioinspirada trouxe melhores resultados.

Neste trabalho, pretendemos desenvolver um modelo diferente que atenda à nutrição comportamental, começando pela minimização do desvio calórico penalizando desvios nutricionais [11], e então aprimorando a formulação com inclusão da divisão de horários e a otimização dinâmica, possibilitando as interações com o usuário. As entradas recebidas são usadas pelo Filtro Colaborativo [12] para prever as preferências alimentares.

\section{MÉtodos E MATERIAIS}

O modelo desenvolvido neste estudo considera de 1 a 3 porções de uma receita e uma meta calórica diária de 2.000 Kcal, conforme recomendação média para um adulto brasileiro [13]. Além das calorias, o modelo inclui outros 10 nutrientes: proteínas (PT), lipídios (LI), carboidratos $(\mathrm{CH})$, magnésio $(\mathrm{Mg})$, ferro $(\mathrm{Fe})$, fósforo $(\mathrm{Fs})$, cálcio $(\mathrm{Ca})$, fibras (FI ), zinco (Zi) e manganês (Mn). A Tabela I apresenta as recomendações de ingestão diária dos nutrientes $[14 ; 15]$.

\begin{tabular}{ccc|ccc} 
Mínimo & Nutriente & Máximo & Mínimo & Nutriente & Máximo \\
\hline 150 & $\leq C H \leq$ & 300 & 1000 & $\leq C a \leq$ & 2500 \\
25 & $\leq F I \leq$ & - & 14 & $\leq F e \leq$ & 45 \\
45 & $\leq L I \leq$ & 98 & 7 & $\leq Z n \leq$ & 34 \\
75 & $\leq P T \leq$ & 210 & 700 & $\leq F s \leq$ & 4000 \\
& & 260 & $\leq M g \leq$ & 350 \\
& & 2,3 & $\leq M n \leq$ & - \\
\hline & & &
\end{tabular}

INGESTÃO NUTRICIONAL DIÁRIA RECOMENDADA [14;15].

Idealmente, um plano alimentar deve distribuir as calorias ao longo do dia e não apenas em uma, duas ou três refeições. Isto é: café da manhã, almoço e jantar, pois, tendemos a comer mais, quando os intervalos entre refeições são iguais ou maiores do que 4-5 horas [16]. Portanto, é imprescindível se alimentar entre as refeições principais, motivo pelo qual dividimos o plano de refeições conforme mostrado na Figura 1 .

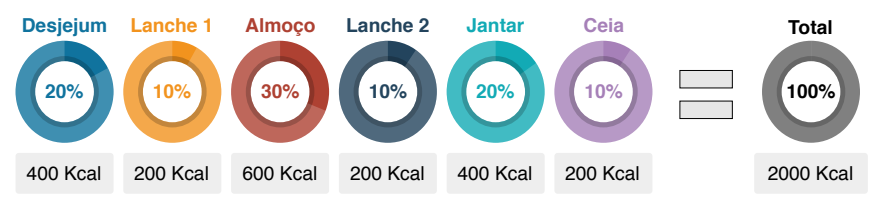

Figura 1. Divisão calórica das refeições.

Para evitar a concentração de apenas um nutriente em uma determinada refeição, a divisão calórica considera também o equilíbrio nutricional e não apenas o valor calórico. A função de avaliação (FA) é mostrada na Equação 1, esta ultima respeitando as restrições das Equações 2, 3 e 4.

$$
\min \left|2000^{2}-\left(\sum_{i} \sum_{j} k c a l_{i_{j}} y_{i_{j}}\right)^{2}\right|, \forall i \in H, j \in T
$$

$$
\begin{gathered}
\sum_{i} \sum_{j} \sum_{k} m_{i_{j k}} y_{i_{j}} \geq b_{k}, \forall i \in H, j \in T, k \in N \\
\sum_{i} \sum_{j} \sum_{k} m_{i_{j_{k}}} y_{i_{j}} \leq B_{k}, \forall i \in H, j \in T, k \in N \\
y_{i_{j}} \in\{0,3\}, \forall i \in T
\end{gathered}
$$

- $H, T$ e $N$ representam a quantidade de refeições, conjunto de alimentos disponíveis e nutrientes considerados no problema, respectivamente.

- A formulação matemática é definida por números inteiros, de 0 a 3 , representados pela variável de decisão $y$, onde $y_{i_{j}}=1$ representa a inclusão de uma porção de alimento $j \in T$ na refeição $i \in H$ da dieta.

- Além disso, $m_{j_{k}}$ representa a quantidade de nutrientes $k \in N$ que está contida na porção do alimento $j \in T$ com $b_{k}$ e $B_{k}$ sendo os requisitos mínimos e máximos para o nutriente $k \in N$.

- A variável $k_{c a l} l_{i_{j}}$ representa a contagem de calorias para o alimento ou receita $j \in T$ para a refeição do horário $i \in H$.

O processo de avaliação das refeições deve respeitar os limites inferiores (LB) e superiores (UB) de cada nutriente, na proporção do percentual calórico esperado para aquela refeição. Quando ocorre uma violação, um mecanismo baseado em penalidades é acionado. Desta forma, o mecanismo de penalidades [11], mostrado na Equação 5, é adicionado à função de aptidão, resultando na Equação 6.

$$
\begin{aligned}
& \sum_{k} \frac{\left(\left|\sum j_{\in T_{i}}\left(m_{i_{j_{k}}} y_{i_{j}}\right)-b_{i_{k}}\right|\right)}{b_{i_{k}}} \cdot M \\
& \min \left|2000^{2}-\left(\sum_{i} \sum_{j} k_{c a l} i_{i_{j}} y_{i_{j}}\right)^{2}\right|+\sum_{i} \sum_{k} \frac{\left(\left|\sum_{j} \epsilon_{T i}\left(m_{i_{j_{k}}} y_{i_{j}}\right)-b_{i_{k}}\right|\right)}{b_{i_{k}}} \cdot M
\end{aligned}
$$

A variável $M$ é um número constante, de valor grande, usada como taxa de penalidade para restrições violadas, este valor regula a intensidade da penalidade, que pode diferir para cada nutriente. Neste trabalho as penalidades têm pesos iguais e proporcionais para todos os nutrientes. Os outros termos são os mesmos mencionados nas Equações 1, 2, 3 e 4.

\section{A. Interações de usuário por meio de otimização dinâmica}

Quando temos um comportamento estático, os valores ótimos são invariáveis, permanecendo na mesma posição durante o processo de busca. Ao incluir a otimização dinâmica, por meio das interações do usuário, o problema passa a variar em suas propriedades e função objetivo, de forma que o ótimo se desloca para outras posições no espaço de busca. Na Figura 2 ilustramos o ciclo interativo realizado por um usuário ao longo do dia.

Após uma interação do usuário, o total de calorias obtidas é comparado com o cardápio inicialmente proposto para aquele horário, a diferença calórica, se houver, é calculada pela Equação 7, onde o valor $i$ indica o horário das refeições 


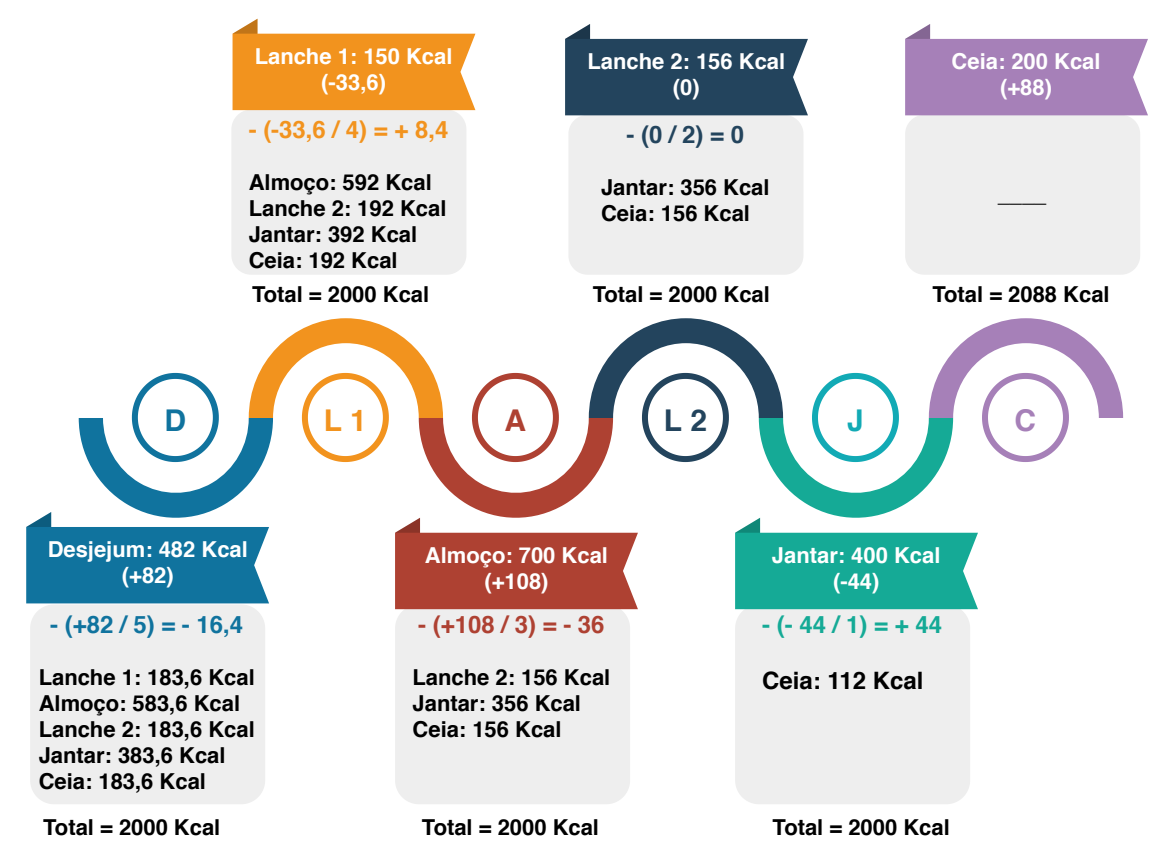

Figura 2. Interação dinâmica.

que está relacionado ao número de calorias esperado naquele horário.

$$
\operatorname{dif}_{i}=\sum_{j=1} k c a l_{i_{j}} y_{i_{j}}-\text { KcalMeta }_{i}
$$

onde $k_{c a l} i_{i_{j}}$ representa o valor obtido das calorias na receita $j$ do horário $i$, enquanto KcalMeta $_{i}$ representa o valor de calorias esperado para a refeição $i$.

Os desvios calóricos promovidos pela diferença de ingestão do usuário são recalculados e compensados nas próximas refeições. O número de refeições restantes é utilizado parra calcular, conforme Equação 8, o quanto de calorias será modificado nas próximas refeições.

$$
a=\frac{d i f_{1}}{I-i}
$$

Os valores $a, I$ e $i$ representam as calorias que serão adicionadas ou diminuídas para as próximas refeições, o número total de refeições e a refeição atual, respectivamente. O cardápio gerado inicialmente possui livre escolha dos alimentos em todo o espaço de busca disponível. Assim que ocorre a primeira entrada do usuário, os itens consumidos são comparados com o histórico de consumo de outras pessoas para previsão de futuras refeições.

Na Figura 2, anteriormente ilustrada, a primeira interação do usuário com o sistema, café da manhã, foi diferente da proposta anterior, ultrapassando em $82 \mathrm{Kcal}$ o esperado. Esta diferença aciona o mecanismo compensatório, o qual gera um novo cardápio, modificando as próximas refeições. O mesmo ocorre para o lanche da manhã (L1), almoço (A) e o jantar
(J). Para o lanche da tarde (L2) a entrada do usuário foi a mesma proposta, não acionando o mecanismo compensatório, mantendo assim o cardápio. A última refeição (C) não ativa o mecanismo compensatório porque não existem outras refeições para compensar a diferença final de $88 \mathrm{Kcal}$.

\section{B. Predição de preferências por filtro colaborativo}

Filtro colaborativo (CF) é uma técnica usada por sistemas de recomendação. A motivação para usar $\mathrm{FC}$ vem da ideia de que as pessoas geralmente recebem boas recomendações de outras com gostos semelhantes [17]. As previsões são específicas para um usuário, mas usam informações coletadas de muitos outros usuários. Este trabalho utiliza o filtro colaborativo baseado na similaridade entre os itens [18; 19].

Inicialmente temos uma matriz esparsa contendo os usuários e alimentos $(U \times I)$, com quantidades ingeridas, entre 0 e 3 , em cada célula. Em seguida, começamos com o cálculo das relações entre os itens, com objetivo final de construir uma nova matriz de itens $\mathrm{x}$ itens contendo os pesos de cada relação, entre cada um dos alimentos, onde uma correlação perfeita é igual a 1 e nenhuma correlação é igual a 0 .

Esta relação entre dois itens é baseada na similaridade de cossenos, onde, dados dois vetores de alimentos ingeridos, $A$ e $B$, para uma dada refeição $i$, a similaridade entre cosseno, $\cos (\theta)$, é representada usando o produto escalar e sua magnitude conforme mostrado na Equação 9.

$$
\operatorname{sim}_{i}=\cos (\theta)=\frac{A_{i} \cdot B_{i}}{\left\|A_{i}\right\| \cdot\left\|B_{i}\right\|}=\frac{\sum_{j} A_{i_{j}} B_{i_{j}}}{\sum_{j} \sqrt{A_{i_{j}^{2}}} \sum_{j} \sqrt{B_{i_{j}^{2}}}}
$$


Outro aspecto importante é a normalização da lista de itens, de tal maneira que usuários com muitas preferências, isto é, maior diversidade de alimentos ingeridos na tabela, contribuam menos na pontuação. Isto significa que um usuário que gosta de apenas alguns poucos itens, é mais valiosos do que outro que goste de tudo. Este processo é feito calculando a raiz quadrada da soma dos quadrados de todas as classificações, como na Equação 10.

$$
\text { magnitude }_{i}=\sqrt{u_{1}^{2}+u_{2}^{2}+u_{3}^{2}+\ldots}
$$

Com o valor de magnitude m $_{i}$ um novo vetor, conforme Equação 11, é criado, onde obtemos valores entre 0 e 1, representando a importância das preferências de cada usuário.

$$
i m p_{i}=\left(\frac{u_{i_{1}}}{\text { magnitude }_{i}}, \frac{u_{i_{2}}}{\text { magnitude }_{i}}, \frac{u_{i_{3}}}{\text { magnitude }_{i}}, \ldots\right)
$$

Com a matriz de similaridade pronta, a Equação 12 calcula a pontuação de cada item classificado, onde a pontuação do usuário $u$, item $j$ na refeição $i$ é obtida somando todos os pesos para este alimento naquela refeição $W_{i_{j}}$, multiplicado pela classificação de importância do usuário para o item daquela refeição $r_{u_{i_{j}}}$. Em seguida, dividimos pela soma de todos os pesos deste alimento naquela refeição $W_{i_{j}}$.

$$
\operatorname{score}(u, i, j)=\frac{\sum_{j} W_{i_{j}} r_{u_{i_{j}}}}{\sum_{j} W_{i_{j}}}
$$

Por fim, a inclusão desta lista de itens pontuados, referente ao usuário com itens mais semelhantes aos até então ingeridos, baseia-se no método decrescente de primeiro ajuste (First Fit Decreasing), até um determinado limite de porcentagem calórica. Este limite é necessário para que o algoritmo ainda tenha uma porcentagem de calorias livres para reequilibrar, em um mesmo horário de refeição, possíveis tendências a desvios nutricionais provocados após inserções das preferências.

\section{Base de dados}

Os nutrientes para cada alimento utilizado neste trabalho foram obtidos da tabela brasileira de composição dos alimentos (TACO) [20]. Esta tabela contém a composição de 568 alimentos separados por categorias. O tamanho da porção padrão do TACO foi modificado com base na categoria, conforme mostrado na Tabela II

\begin{tabular}{ccc} 
Categoria & Intervalo & Tamanho da porção \\
\hline Amêndoas & $1-11$ & $20 \mathrm{~g}$ \\
Bebidas & $12-27$ & $200 \mathrm{ml}$ \\
Carboidratos Simples & $28-89$ & $30 \mathrm{~g}$ \\
Carboidratos Compostos & $90-137$ & $100 \mathrm{~g}$ \\
Frutas & $138-234$ & $100 \mathrm{~g}$ \\
Grãos & $235-268$ & $50 \mathrm{~g}$ \\
Lácteos & $269-292$ & $50 \mathrm{~g}$ \\
Óleos & $293-306$ & $10 \mathrm{~g}$ \\
Carnes e peixes & $307-492$ & $100 \mathrm{~g}$ \\
Vegetais & $493-568$ & $80 \mathrm{~g}$ \\
\hline
\end{tabular}

TAMANHO DE PORÇÃO POR CATEGORIA.

\section{Algoritmo Evolucionário}

O algoritmo bioinspirado utilizado possui três principais aspectos da teoria da evolução [21], são eles o cruzamento $(i)$, a mutação (ii) e seleção (iii). Estes três aspectos, modelados como operadores, são aplicados iterativamente a partir de uma população inicial até que o critério de parada seja alcançado.

Dada uma população $X=x_{1}, x_{2}, \ldots, x_{p_{o_{p}}}$, onde $x_{i}$ é o $i$ indivíduo de $X$. Seja $x$ a população $X^{g}$ na geração $g$. Desta forma, temos que a população inicial está na primeira geração. No final da iteração, diz-se que a população resultante $X^{g}+1$ está na geração $g+1$. A população inicial $X=x_{1}, x_{2}, \ldots, x_{p_{o_{p}}}$ é gerada aleatoriamente com $|X|=P_{o_{p}}$ indivíduos. A representação do indivíduo consiste em um vetor de inteiros, onde cada posição representa um alimento diferente, contendo um valor de 0 a 3 , representando o número de porções de alimentos incluídas no cardápio.

Quanto aos operadores, o cruzamento é o primeiro operador aplicado em cada geração. Este operador gera pares de novas soluções, por meio da combinação de dois indivíduos selecionados da população atual. Cada par de soluções selecionadas é combinado para gerar novos indivíduos, respeitando a probabilidade de cruzamento de $T_{c}$. O cruzamento de um ponto [22] foi o operador de recombinação utilizado. Ele funciona escolhendo um número aleatório no intervalo (sendo 1 , o comprimento da codificação/representação), e então dividindo ambos os pais neste ponto e criando os dois filhos trocando as caudas.

O operador de mutação é aplicado ao vetor de solução. Ele gera um novo valor para cada posição com uma probabilidade $T_{m}$ configurável. Por fim, o operador de seleção escolhe os indivíduos que serão submetidos à próxima geração. $\mathrm{O}$ resumo das etapas realizadas pode ser visto no fluxograma da Figura 3.

\section{Resultados E Discussões}

Os experimentos computacionais foram realizados na $G o$ ogle Cloud Platform em servidores categoria e2-medium (2 vCPUs, 4 GB memória) com uma vCPU Intel Haswell, executando apenas Linux Ubuntu Server 20.04 sem interface gráfica instalada. Os algoritmos foram implementados em $\mathrm{C}++$. Foram executadas 30 réplicas por experimento.

Os parâmetros do algoritmo bioinspirado utilizado foram escolhidos após uma breve comparação, conforme mostrado na Tabela III, onde se consideram taxas de cruzamento fixadas em 0,6 , mutação em 0,4 , tamanho da população em 100, número de gerações em 2500, a proporção elitista de 0,02, mutação do tipo random reseting e o operador de torneio para seleção de pais e filhos, este ultimo, com propriedades que não requer nenhum conhecimento global da população. Em vez disso, o operador depende apenas de uma relação de ordenação que possa comparar e classificar quaisquer dois indivíduos. 


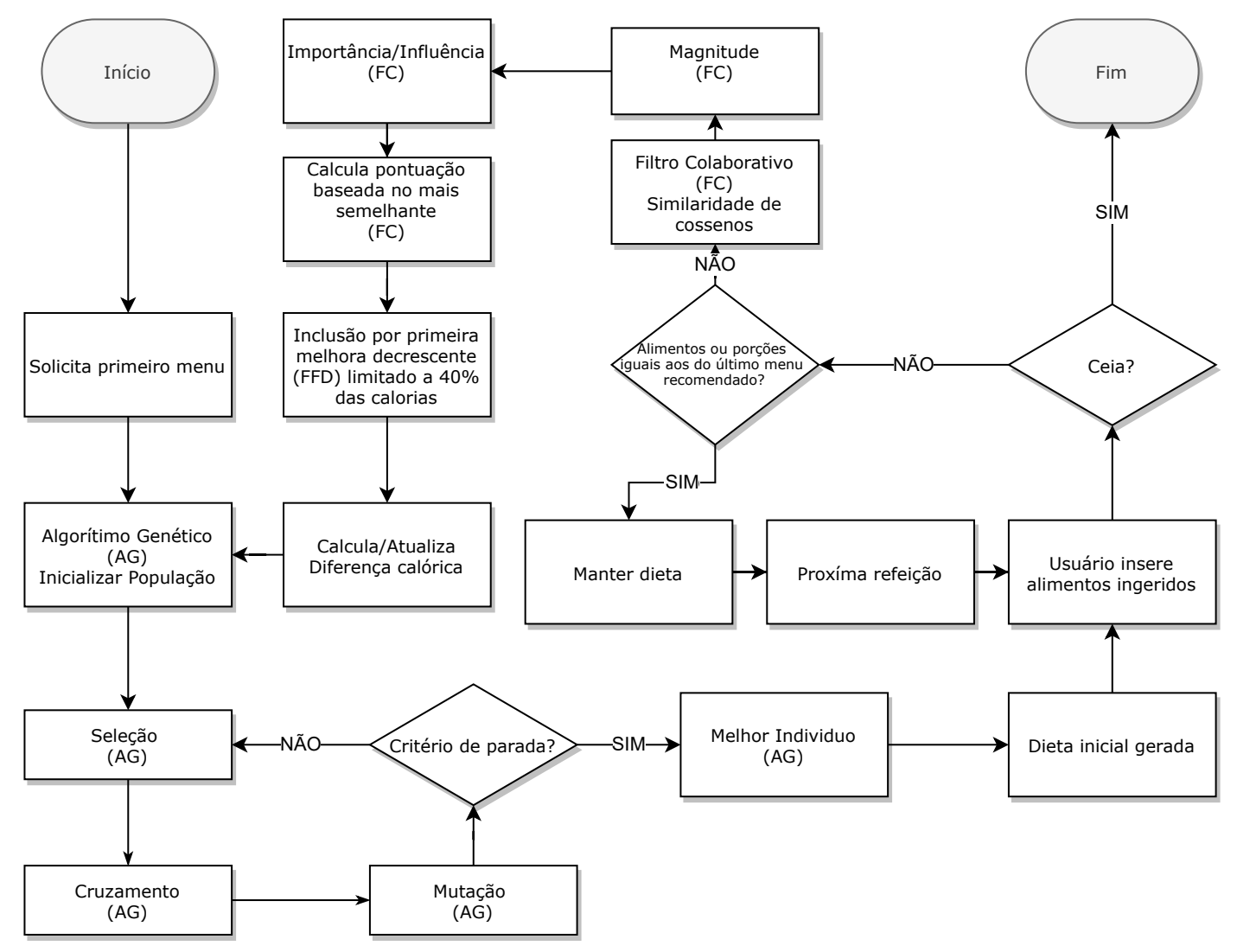

Figura 3. Fluxograma do modelo proposto.

\begin{tabular}{c|cc} 
seleção & função objetivo & calorias \\
\hline uniforme & $8,82 \mathrm{E}+13$ & 93909 \\
torneio & $\mathbf{1 3 3 3 , 2 2}$ & $\mathbf{2 0 0 0}$ \\
roleta & $7,62 \mathrm{E}+14$ & 87325 \\
sus & $4,10 \mathrm{E}+14$ & 69843 \\
overselection & 2913,66 & 2000,1 \\
torneio round robin & $2,44 \mathrm{E}+12$ & 5324 \\
\hline
\end{tabular}

TABELA COMPaRATIVA ENTRE MÉTODOS DE SELEÇÃO.

Os resultados obtidos a partir de uma única refeição estática reproduzida são mostrados na Tabela IV.

\begin{tabular}{c|cc} 
Nome & Média & Desvio Padrão \\
\hline Função objetivo & $2,96 \times 10^{05}$ & $3,98 \times 10^{05}$ \\
Calorias(g) & $2,00 \times 10^{03}$ & $3,05 \times 10^{01}$ \\
Proteínas(g) & $1,05 \times 10^{02}$ & $2,47 \times 10^{01}$ \\
Carboidratos(g) & $3,21 \times 10^{02}$ & $3,23 \times 10^{01}$ \\
Fibras(g) & $8,58 \times 10^{01}$ & $1,16 \times 10^{01}$ \\
Gorduras(g) & $5,19 \times 10^{01}$ & $9,46 \times 10^{00}$ \\
Cálcio(mg) & $1,56 \times 10^{03}$ & $3,64 \times 10^{02}$ \\
Magnésio(mg) & $6,74 \times 10^{02}$ & $1,14 \times 10^{02}$ \\
Fosforo(mg)* & $1,79 \times 10^{03}$ & $3,58 \times 10^{02}$ \\
Ferro(mg) & $2,38 \times 10^{01}$ & $5,29 \times 10^{00}$ \\
Zinco(mg) & $1,49 \times 10^{01}$ & $2,83 \times 10^{00}$ \\
Manganés (mg) & $1,28 \times 10^{01}$ & $5,28 \times 10^{00}$ \\
\hline \multicolumn{3}{|c}{ Tabela IV } \\
\multicolumn{3}{c}{ ModELO ESTÁTICO DE REFEIÇÃO ÚNICA. }
\end{tabular}

A Figura 4 mostra que parte dos cardápios gerados pos- sui um pequeno excesso de carboidratos, enquanto todos os cardápios ultrapassaram o limite superior de magnésio.

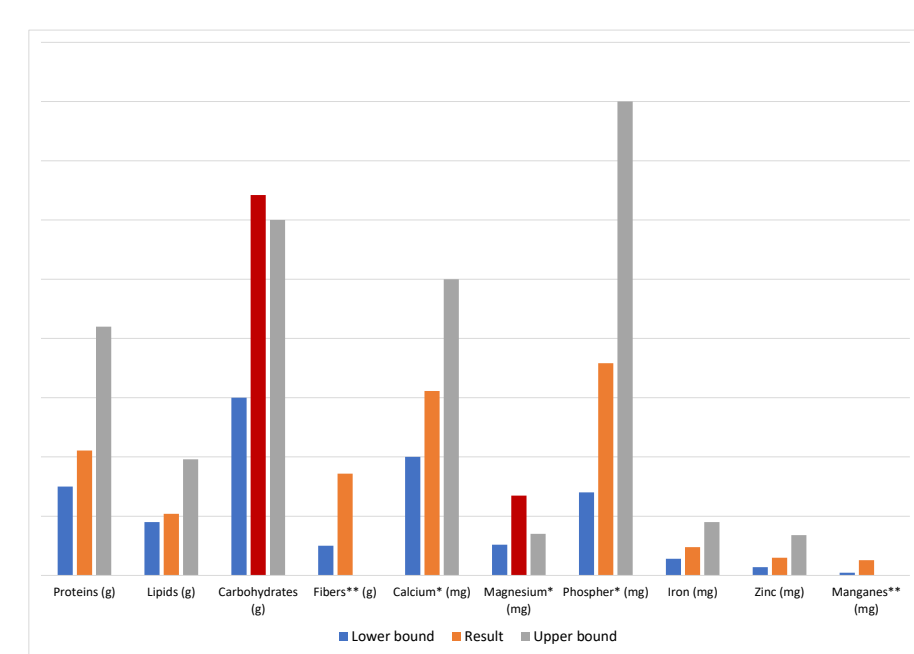

Figura 4. Desvio nutricional do modelo estático de refeição única.

A inclusão dos horários, conforme apresentado na Tabela V, gerou um desvio calórico em refeições menores, como lanches 
(142 kcal e $146 \mathrm{kcal})$ e ceia (164 kcal), além disso, o tempo para conclusão foi 5 vezes maior. disponíveis para equilibrar a refeição após a inclusão das preferências.

\begin{tabular}{|c|c|c|c|c|c|c|c|c|c|c|c|c|}
\hline & \multicolumn{2}{|c|}{ Desjejum } & \multicolumn{2}{|c|}{ Lanche 1} & \multicolumn{2}{|c|}{ Almoço } & \multicolumn{2}{|c|}{ Lanche 2} & \multicolumn{2}{|c|}{ Jantar } & \multicolumn{2}{|c|}{ Ceia } \\
\hline Nome & AVG & +- & AVG & +- & AVG & +- & AVG & +- & AVG & $\mathbf{v}$ & AVG & +- \\
\hline Função Objetivo &, $56 \times 10^{5}$ & $2,22 \times 10^{5}$ & $9,15 \times 10^{4}$ & $6,25 \times 10^{4}$ & $2,76 \times 10^{5}$ & $1,17 \times 10^{5}$ & $1,02 \times 10^{5}$ & $7,71 \times 10^{4}$ & $1,90 \times 10^{5}$ & $1,97 \times 10^{5}$ & $9,87 \times 10^{4}$ & $5,71 \times 10^{4}$ \\
\hline Calorias & $4,10 \times 10^{2}$ & $2,55 \times 10^{1}$ & $42 \times 10^{2}$ & $8,91 \times 10^{1}$ & $6,01 \times 10^{2}$ & $3,66 \times 10^{-02}$ & $3,46 \times 10^{2}$ & $7,85 \times 10^{1}$ & $4,07 \times 10^{2}$ & $1,73 \times 10^{1}$ & $3,64 \times 10^{2}$ & $8,35 \times 10^{1}$ \\
\hline $\mathrm{Pr}$ & $40 \times 10^{1}$ & $6,59 \times 10^{0}$ & $03 \times 10^{1}$ & $6,35 \times 10^{0}$ & $3,19 \times 10^{1}$ & $1,19 \times 10^{1}$ & $2,07 \times 10^{1}$ & $5,37 \times 10^{0}$ & $2,17 \times 10^{1}$ & $5,35 \times 10^{0}$ & $2,20 \times 10^{1}$ & $8,92 \times 10^{0}$ \\
\hline Carbo & $57 \times 10^{1}$ & $7,16 \times 10^{0}$ & $6,82 \times 10^{1}$ & $1,89 \times 10^{1}$ & $1,15 \times 10^{2}$ & $1,95 \times 10^{1}$ & $7,03 \times 10^{1}$ & $1,71 \times 10^{1}$ & $8,13 \times 10^{1}$ & $8,09 \times 10^{0}$ & $7,27 \times 10^{1}$ & $1,66 \times 10^{1}$ \\
\hline Go & $16 \times 10^{0}$ & $1,16 \times 10^{0}$ & $4,57 \times 10^{0}$ & $2,57 \times 10^{0}$ & $9,69 \times 10^{0}$ & $4,17 \times 10^{0}$ & $4,26 \times 10^{0}$ & $2,03 \times 10^{0}$ & $5,83 \times 10^{0}$ & $3,88 \times 10^{0}$ & $4,30 \times 10^{0}$ & $1,87 \times 10^{0}$ \\
\hline & $5 \times 10^{2}$ & $1,66 \times 10^{2}$ & $5,74 \times 10^{2}$ & $2,60 \times 10^{2}$ & $6,98 \times 10^{2}$ & $1,73 \times$ & $6,27 \times 10^{2}$ & $2,54 \times 10^{2}$ & $5,69 \times 10^{2}$ & $2,32 \times 10^{2}$ & $5,98 \times 10^{2}$ & $2,28 \times 10^{2}$ \\
\hline & $47 \times 10^{2}$ & $4,71 \times 10^{1}$ & $2,38 \times 10^{2}$ & $8,35 \times 10^{1}$ & 2,76 & $6,86 \times$ & $2,57 \times 10^{2}$ & $1,06 \times 10^{2}$ & $2,33 \times 10^{2}$ & $7,48 \times 10^{1}$ & $2,47 \times 10^{2}$ & $9,84 \times 10^{1}$ \\
\hline & $2 \times$ & $1,18 \times$ & $07 \times 10^{2}$ & $1,22 \times$ & & & 4,23 & $1,05>$ & & $1,00 \times 10^{2}$ & & $1,58 \times 10^{2}$ \\
\hline $\mathrm{Fe}$ & $47 \times 10^{0}$ & $3,17 \times 10^{0}$ & $7,12 \times 10^{0}$ & $3,56 \times 10^{0}$ & $9,62 \times 10^{0}$ & $3,10 \times 10^{0}$ & $8,06 \times 10^{0}$ & $3,45 \times 10^{0}$ & $8,42 \times 10^{0}$ & $2,68 \times 10^{0}$ & $6,98 \times 10^{0}$ & $2,92 \times 10^{0}$ \\
\hline Manganês & $5,41 \times 10^{0}$ & $3,82 \times 10^{0}$ & $5,62 \times 10^{0}$ & $4,27 \times 10^{0}$ & $4,52 \times 10^{0}$ & $3,37 \times 10^{0}$ & $6,15 \times 10^{0}$ & $4,64 \times 10^{0}$ & $4,93 \times 10^{0}$ & $4,60 \times 10^{0}$ & $5,92 \times 10^{0}$ & $4,38 \times 10^{0}$ \\
\hline
\end{tabular}

EXPERIMENTOS COM DIVISÃO DE HORÁRIOS.

Neste ponto, nosso modelo apresenta resultado calórico total com variação de $470 \mathrm{Kcal}$ acima da meta de $2000 \mathrm{Kcal}$, enquanto o trabalho de [11], traz uma diferença semelhante de $446 \mathrm{Kcal}$ negativas, em relação à meta de $2000 \mathrm{kcal}$.

Ao incluirmos a otimização dinâmica, buscamos avaliar a capacidade do algoritmo em fazer o cálculo correto e gerar um novo menu atendendo a essa nova meta calórica. Neste sentido, o sistema conseguiu realizar o cálculo compensatório correto desde que o limite calórico do dia não seja ultrapassado em uma única refeição, por exemplo, consumir mais de 2.000 kcal no café da manhã. Considerando o cardápio reequilibrado, apresentado na Tabela VI, é possível identificar um maior sucesso no reequilíbrio das dietas para almoço e jantar.

\begin{tabular}{c|ccccc} 
horários & Lanche 1(avg +-) & Almoço & Lanche 2 & Jantar & Ceia \\
\hline Café da manhã & 33284 & 5990.6 & 327137 & 41647 & 308145 \\
Lanche 2 & - & 5990.4 & 329113 & 40413 & 330123 \\
Almoço & - & - & 279110 & 41257 & 256108 \\
Lanche 2 & - & - & - & 40630 & 26464 \\
Jantar & - & - & - & - & 261154 \\
\hline
\end{tabular}

REBALANCEAMENTO DE DESVIO CALÓRICO.

O valor médio é baseado na diferença obtida entre a nova meta calculada e a gerada no novo menu.

Por fim, para o último modelo considerando preferências, tivemos a limitação de avaliar sua eficiência em relação apenas às categorias de alimentos. $\mathrm{O}$ gerador de instâncias aleatórias utilizou o princípio demonstrado na Tabela VII.

\begin{tabular}{c|c} 
refeição & categorias \\
\hline desjejum & lácteo, frutas, carboidrato simples \\
lanche manhã & amêndoas, frutas, óleos \\
almoço & proteína, carboidrato complexo, bebidas, grãos, vegetais \\
lanche tarde & amêndoas, frutas, óleos \\
jantar & proteína, carboidrato complexo, bebidas, grãos, vegetais \\
ceia & amêndoas, carboidrato simples \\
\hline
\end{tabular}

DIVISÃO DE CATEGORIAS NA GERAÇÃO DO HISTÓRICO ALIMENTAR.

Para medir o impacto da inclusão das preferências e da capacidade de equilíbrio, foi estabelecido o limite e $40 \%$, onde este percentual indica quantas calorias de uma determinada refeição estariam disponíveis para inclusão das preferências classificadas. Os $60 \%$ restantes seriam a quantidade de calorias

\begin{tabular}{c|ccccc} 
& Lanche 1 & Almoço & Lanche 2 & Jantar & Ceia \\
\hline Média & 386,2 & 707,4 & 418,4 & 553,8 & 373,2 \\
Desvio padrão & 93,55 & 59,71 & 69,07 & 86,86 & 79,56 \\
\hline \multicolumn{5}{c}{ Tabela VIII } \\
\hline
\end{tabular}

CALORIAS POR REFEIÇÃo APÓS INCLUSÃO DAS PREFERÊNCIAS.

Em geral, algum item da categoria preferida para aquele horário de refeição esteve presente em $81 \%$ dos cardápios gerados. Amêndoas foi a categoria com menor ocorrência de inclusão, inferior a $30 \%$, mesmo com pontuação considerável. A Figura 5 faz uma comparação do impacto calórico relacionado à inclusão das preferências.

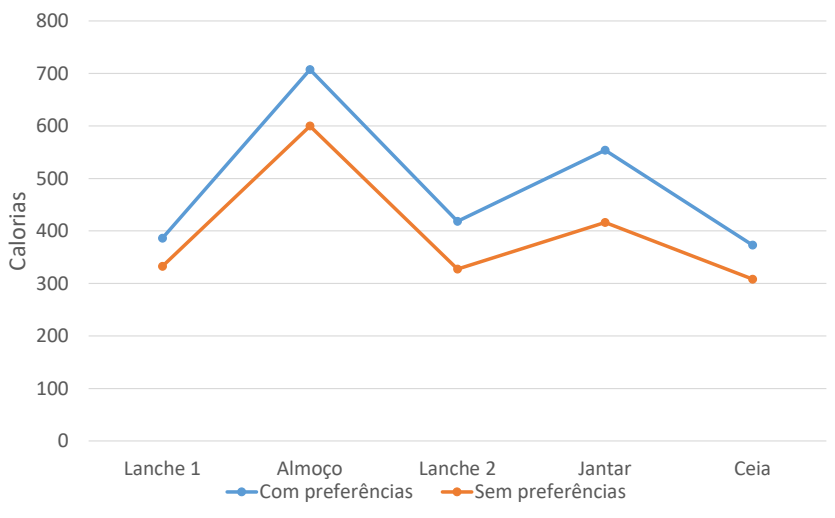

Figura 5. Impacto calórico após inclusão das preferências.

As contribuições deste artigo podem ser resumidas da seguinte forma. Primeiramente, reproduzimos o modelo estático de refeição única. Depois adicionamos ao modelo as divisões de horários das refeições. Posteriormente, desenvolvemos o modelo interativo, avaliando sua eficácia e limitações em reequilibrar o cardápio de acordo com o consumo das refeições do usuário. Por fim, incluímos o modelo de predição de preferências baseado em filtro colaborativo, limitando a inclusão dos itens mais bem classificados em $40 \%$ das calorias estimadas consumidas para cada refeição, avaliando o impacto da inclusão de preferências na dieta. 


\section{CONCLUSÃO E TRABALhOS FUTUROS}

O objetivo deste trabalho foi uma melhoria nos modelos PD atuais com a inclusão das iterações e predição de preferências. Tendo em vista que foi utilizada uma base de dados alimentar real, contendo todos os alimentos existentes em sua forma integral, pode ser interessante considerar, em pesquisas futuras, o fato relacionado ao excesso de manganês presente em todos os cardápios gerados, de modo a desenvolver técnicas que busquem atender a todos os aspectos nutricionais sem exceder o nível de manganês. A construção de um histórico alimentar real também seria interessante para avaliar a capacidade de predição, considerando os itens especificamente. Por fim, outro ponto seria ampliar, por meio da recomendação de exercícios físicos, a capacidade do sistema interativo de re-balancear as dietas.

\section{AGRADECIMENTOS}

Os autores agradecem a Universidade Federal de Ouro Preto (UFOP), ao Conselho Nacional de Desenvolvimento Científico e Tecnológico (CNPq - 402956/2016-8), e à Fundação de Amparo à Pesquisa do estado de Minas Gerais (FAPEMIG APQ-00040-14) pelo apoio ao desenvolvimento deste trabalho.

\section{REFERÊNCIAS}

[1] G. J. Stigler, "The cost of subsistence," Journal of farm economics, vol. 27, no. 2, pp. 303-314, 1945.

[2] L. M. Lancaster, "The evolution of the diet model in managing food systems," Interfaces, vol. 22, no. 5, pp. 59-68, 1992.

[3] A. R. Job and A. C. de Oliveira Soares, "Percepção dos acadêmicos de nutrição sobre a efetividade a longo prazo dos métodos de dietas restritivas para a perda e o controle de peso," RBONE-Revista Brasileira de Obesidade, Nutrição e Emagrecimento, vol. 13, no. 78, pp. 291-298, 2019.

[4] A. V. Sarraceni and G. d. S. F. Donha, "Percepção de nutricionistas a respeito da prática clínica embasada na nutrição comportamental," 2018.

[5] F. Bernardi, C. Cichelero, and M. R. Vitolo, "Comportamento de restrição alimentar e obesidade," Revista de Nutrição, vol. 18, no. 1, pp. 85-93, 2005.

[6] M. Alvarenga, C. Antonaccio, F. Timerman, and M. Figueiredo, Nutrição comportamental. Editora Manole, 2015.

[7] J. Freyne and S. Berkovsky, "Intelligent food planning: personalized recipe recommendation," in Proceedings of the 15th international conference on Intelligent user interfaces, 2010, pp. 321-324.

[8] T. N. T. Tran, M. Atas, A. Felfernig, and M. Stettinger, "An overview of recommender systems in the healthy food domain," Journal of Intelligent Information Systems, vol. 50, no. 3, pp. 501-526, 2018.
[9] C. Trattner and D. Elsweiler, "Food recommender systems: important contributions, challenges and future research directions," arXiv preprint arXiv:1711.02760, 2017.

[10] J. G. R. Silva, I. A. Carvalho, M. M. S. Loureiro, V. da Fonseca Vieira, and C. R. Xavier, "Developing tasty calorie restricted diets using a differential evolution algorithm," in International Conference on Computational Science and Its Applications. Springer, 2016, pp. 171-186.

[11] J. G. R. Silva, M. Gerais, H. J. C. Barbosa, I. A. Carvalho, V. da Fonseca Vieira, and C. R. Xavier, "Um estudo comparativo entre um algoritmo gen etico e um algoritmo de evoluc ao diferencial para o problema da dieta restrita em calorias," 2017.

[12] J. L. Herlocker, J. A. Konstan, L. G. Terveen, and J. T. Riedl, "Evaluating collaborative filtering recommender systems," ACM Transactions on Information Systems (TOIS), vol. 22, no. 1, pp. 5-53, 2004.

[13] MS, "Guia alimentar para a população brasileira: promovendo a alimentação saudável," 2006.

[14] S. ANVISA, "Agência nacional de vigilância sanitária," 2014.

[15] F.-t. A. Fortificados, S. C. de Nutrição, I. Brasil, N. A. G. de França, and L. A. Martini, "Funções plenamente reconhecidas de nutrientes," 2014.

[16] M. J. Munsters and W. H. Saris, "Effects of meal frequency on metabolic profiles and substrate partitioning in lean healthy males," PloS one, vol. 7, no. 6, p. e38632, 2012.

[17] D. Billsus, M. J. Pazzani et al., "Learning collaborative information filters." in Icml, vol. 98, 1998, pp. 46-54.

[18] C. Miranda and A. Jorge, "Incremental collaborative filtering for binary ratings," 12 2008, pp. 389-392.

[19] M. Sunitha and T. Adilakshmi, "Music recommendation system with user-based and item-based collaborative filtering technique," in Networking Communication and Data Knowledge Engineering. Springer, 2018, pp. 267278.

[20] N. UNICAMP, "Tabela brasileira de composição de alimentos-taco," 2011.

[21] C. Darwin, A. R. Wallace et al., "Evolution by natural selection." Evolution by natural selection., 1958.

[22] J. H. Holland et al., Adaptation in natural and artificial systems: an introductory analysis with applications to biology, control, and artificial intelligence. MIT press, 1992. 